\title{
Disparities in outcomes associated with rural-urban insurance status in China among inpatient women with stroke: a registry-based cohort study
}

\author{
Hong-Qiu Gu ${ }^{1,2}$, Xin Yang ${ }^{1,2}$, Zhen-Zhen Rao ${ }^{3}$, Chun-Juan Wang ${ }^{1,2,4,5,6}$, Xing-Quan Zhao ${ }^{4,6}$, Yi-Long Wang ${ }^{1,4,5,6}$, \\ Li-Ping Liu ${ }^{7}$, Chelsea Liu ${ }^{8}$, Hao $\mathrm{Li}^{1}$, Zi-Xiao $\mathrm{Li}^{1,2,4,6}$, Yong-Jun Wang ${ }^{1,2,4,5,6}$; on behalf of China National \\ Stroke Registries lnvestigators
}

${ }^{1}$ China National Clinical Research Center for Neurological Diseases, ${ }^{2}$ National Center for Healthcare Quality Management in Neurological Diseases, Beijing Tiantan Hospital, Capital Medical University, Beijing 100070, China; ${ }^{3}$ Institute of Molecular Medicine, Yingjie Center, Peking University, Beijing 100871, China; ${ }^{4}$ Vascular Neurology, Department of Neurology, Beijing Tiantan Hospital, Capital Medical University, Beijing 100070, China; ${ }^{5}$ Beijing Key Laboratory of Translational Medicine for Cerebrovascular Disease, Beijing 100053, China; ${ }^{6}$ Center for Stroke, Beijing Institute for Brain Disorders, Beijing 100068, China; ${ }^{7}$ Neuro-intensive Care Unit, Department of Neurology, Beijing Tiantan Hospital, Capital Medical University, Beijing 100070, China; ${ }^{8}$ Johns Hopkins Bloomberg School of Public Health, Johns Hopkins University, Baltimore, USA

Contributions: (I) Conception and design: HQ Gu, ZX Li; (II) Administrative support: ZX Li, H Li; (III) Provision of study materials or patients: X Yang, ZZ Rao; (IV) Collection and assembly of data: HQ Gu, X Yang, ZX Li, CJ Wang; (V) Data analysis and interpretation: HQ Gu, ZX Li, CJ Wang; (VI) Manuscript writing: All authors; (VII) Final approval of manuscript: All authors.

Correspondence to: Zixiao Li. Department of Neurology, Beijing Tiantan Hospital, Capital Medical University, No. 119 Nansihuanxilu, Fengtai District, Beijing 100070, China. Email: lizixiao2008@hotmail.com.

Background: Despite a few studies have demonstrated sex differences in stroke care and outcomes, limited research has explored insurance-related disparities in outcomes, particularly among women stroke patients. The aim was to determine whether rural-urban health insurance status affect the stroke treatment, process of care, and 1-year clinical outcomes for inpatient ischemic stroke in women.

Methods: Women patients with acute ischemic stroke (AIS) covered by New Rural Cooperative Medical Scheme (NRCMS) and urban resident/employee-based basic medical insurance scheme (URBMI/UEBMI) were abstracted from the China National Stroke Registry II (CNSR II). Shared frailty model in the Cox model or generalized estimating equation with consideration of the hospital's cluster effect were used to assess the associations between rural-urban insurance status and quality of care during hospitalization and 1-year stroke outcomes including all-cause death, 1-year recurrence, and 1-year disability.

Results: A total of 5,707 women patients enrolled from 219 hospitals in CNSR II were analyzed. Compared with 2,880 women patients covered by URBMI/UEBMI, 2,827 women patients covered by NRCMS were younger (65.7 versus 68.9 years), less likely to have vascular risk factors, awareness and treatment of hypertension and dyslipidemia prior to stroke. Women covered by NRCMS were more likely to receive early antithrombotics, discharge antithrombotics, lipid-lowering drugs, but less likely to receive antihypertensive medication than those covered by URBMI/UEBMI. One-year all-cause mortality and stroke recurrence were both significantly higher in women patients with NRCMS than those with URBMI/ UEBMI [adjusted hazard ratio (95\% confidence interval): 1.40 (1.06-1.84) and 1.38 (1.04-1.83), separately].

Conclusions: AIS women patients with rural-urban insurance status demonstrated remarkable differences in age, stroke risk factors, awareness and treatment, the process of care, and 1-year stroke recurrence and mortality. Healthcare policymakers need to focus their attention on these disparities and take proper steps to improve primary healthcare service in rural areas. 
Keywords: Ischemic stroke; women; urban; rural; quality of health care; death; recurrence; disability

Submitted May 18, 2019. Accepted for publication Aug 27, 2019.

doi: $10.21037 /$ atm.2019.08.125

View this article at: http://dx.doi.org/10.21037/atm.2019.08.125

\section{Introduction}

Stroke imposes an enormous burden on the health system, with women being disproportionately affected (1). In China, stroke is the third leading cause of death in men, but the second leading cause in women (2). From 1992 to 2012, the incidence of first-ever stroke in rural China grew at a faster annual rate for women $(8.0 \%)$ than for men (5.5\%) (3). Meanwhile, the Chinese Health Statistics for 2010 indicated that stroke is the first and third leading cause of death in rural and urban China (4). In addition, China cardiovascular diseases report 2015 summarized that the projected stroke morality (per 100,000) increased from 110.94 in 2003 to 151.91 in 2014 for rural residents, while increased from 102.44 to 125.78 for urban residents (5). These epidemiological trends suggest an increase in the burden of stroke on women, especially in rural China $(3,6)$.

In rural areas, by the end of June 2012, the New Rural Cooperative Medical Scheme (NRCMS) was administrated by the Chinese government; the scheme covered a budget of 812 million and accounted for over $95 \%$ of the targeted population (7). At present, in urban areas, the Urban Resident Basic Medical Insurance (URBMI) scheme and the Urban Employee Basic Medical Insurance (UEBMI) scheme are in place and cover almost the whole population (8). Populations living in rural China have limited access to medical care than do urban residents along with a larger financial burden, mainly owing to limited funds allocated to the New Rural Cooperative Medical Scheme (NRCMS) (8). Furthermore, compared to women in urban areas, women in rural areas tend to have a low educational level and low income and receive a poor quality of healthcare (2). However, the difference in the stroke burden between women associated with a rural or urban insurance status in China remains unclear. Moreover, China's social health insurance schemes are primarily composed of the NRCMS in rural areas and the URBMI and UEBMI in urban areas (8). Self-pay patients and patients with commercial insurance compose only $8.5 \%$ of the population with stroke.

We investigated how a rural or urban insurance status impacts the demographic and clinical characteristics, stroke risk factor profiles and treatment prior to stroke, the process of care during hospitalization, and 1-year clinical outcomes of acute ischemic stroke (AIS) among women in China who are covered by the NRCMS and URBMI/UEBMI.

\section{Methods}

\section{China National Stroke Registry II Design and Hospital Selection}

We have described the details of the design and conduct of the China National Stroke Registry II (CNSR II) previously $(9,10)$. The CNSR II was sponsored by the National Health Commission. It was a prospective, hospital-based and nationwide registry. The primary objective of the CNSR II is to assess patient clinical features, the delivery of stroke care in clinical practice, and 1-year clinical outcomes. Chinese secondary or tertiary public hospitals can be included in the registry. A total of 219 hospital sites were selected by the steering committee from the China National Network of Stroke Research in our study.

\section{Case enrollment and study population}

This study collected data from June 2012 to January 2013. Patients were recruited from all eligible hospitals in China consecutively. Patients were eligible if they were $\geq 18$ years of age and presented within seven days of the index event of AIS, transient ischemic attack (TIA), spontaneous intracerebral hemorrhage, or subarachnoid hemorrhage confirmed via brain computed tomography or magnetic resonance imaging. All the written informed consent forms were obtained from the patients or their designated relatives.

\section{Data collection and abstraction}

A standardized data collection form was utilized by all participating sites. Participating hospitals nationwide use trained hospital personnel to identify eligible patients, obtain informed consent, and enlist patients for all their stroke hospitalization. The pre-stroke modified Rankin 
Scale (mRS) (with scores ranging from 0 to 6 , with a score of 0 indicating no disability and higher scores indicating more severe disability) (11) and National Institutes of Health Stroke Scale (NIHSS) (with scores ranging from 0 to 42 , with higher scores indicating a more severe deficit) (12) scores were acquired from physical exams by trained neurologists. Other patient data extracted from the medical records included demographics, health insurance status, education, household income, history of smoking and alcohol consumption, vascular risk factors, medication usages prior to stroke, laboratory tests, and discharge status.

\section{Prevalence, unawareness, and treatment of vascular risk factors}

The prevalence, unawareness, and treatment of vascular risk factors including hypertension disease, dyslipidemia, diabetes mellitus, and atrial fibrillation were analyzed. The prevalence of risk factors in our study population prior to stroke was determined by self-reported awareness of hypertension disease, diabetes mellitus, dyslipidemia, and atrial fibrillation, and was defined as the frequency of these chart-confirmed factors in the medical history and by the usage of corresponding medications. The unawareness rate of these four vascular risk factors was calculated as the ratio of initial physician diagnosis during hospitalization to our study population. The treatment medications prescribed prior to the stroke included antiplatelets, antihypertensives, anti-diabetics, and lipid-lowering drugs. Current or previous history of smoking and moderate and heavy alcohol consumption ( $\geq 2$ standard alcohol drinks per day) were also recorded.

\section{Quality measures definitions}

A total of 7 guideline-recommended performance metrics of medication usage were pre-specified for this study based on national recommended guidelines and the Get With The Guidelines-Stroke (GWTG-Stroke) criteria (13-15). These guidelines included: (I) administration of intravenous tissue-type plasminogen activator in patients who arrive within 2 hours after symptom onset and were treated within 3 hours; (II) administration of antithrombotic medication within 48 hours of admission; (III) administration of antithrombotic medication; (IV) administration of anticoagulants for atrial fibrillation; (V) administration of antihypertensive medication for patients with hypertension; (VI) administration of medications for lowering low- density lipoprotein (LDL) levels $\geq 100 \mathrm{mg} / \mathrm{dL}$; and (VII) administration of hypoglycemia medication for diabetes mellitus. The definitions of these performance measures are shown in Table S1.

\section{Clinical outcomes}

Hospital trained personnel who followed standardized scripts to contact patients after the stroke at 3, 6 and 12 months. During the follow-up periods, stroke recurrences associated with re-hospitalization were sourced to the attended hospitals to ensure a reliable diagnosis. In cases of suspected recurrent cerebrovascular events without hospitalization, the decisions were made by the research coordinators together with the principal investigator. Therefore, clinical outcomes analyzed include all-cause death, 1-year stroke recurrence, and disability (defined as $\mathrm{mRS}$ score $>2$ ). A patient was considered as lost to followup if: (I) the consent to participate was withdrawn; (II) at each follow-up time point, contact could not be made after three telephone calls each day in the morning, afternoon, and early evening for one working week. Our analysis was based on patients with complete follow-up information.

\section{Statistical analysis}

Patient clinical characteristics and process of care during hospitalization were compared between patients covered by URBMI/UEBMI and by NRCMS.

Continuous and categorical variables are reported as mean \pm standard deviation (SD) or frequencies (percentages) and were compared using the $t$-test or $\chi^{2}$ test, respectively. According to the Chinese health statistic yearbook, in urban areas, the average family income per capita per month was $1,423 \mathrm{RMB}$ in 2008. In rural areas, the average family income per capita per month was $559 \mathrm{RMB}$ (16). Also, approximately $50 \%$ of the residents, including both urban and rural residents, lived on a family income of $\leq 1,000 \mathrm{RMB}$ per month. Thus, the monthly income per capita was categorized into two groups for our analysis.

The relationship between insurance status and the clinical outcomes, including all-cause death and 1-year stroke recurrence, were further assessed using Cox proportional hazard models. To obtain a parsimonious and stable model, backward selection methods with the default alpha 0.05 were applied to select further covariates from a list of candidate risk factors were applied. Candidate risk factors were clinically plausible variables and selected based 


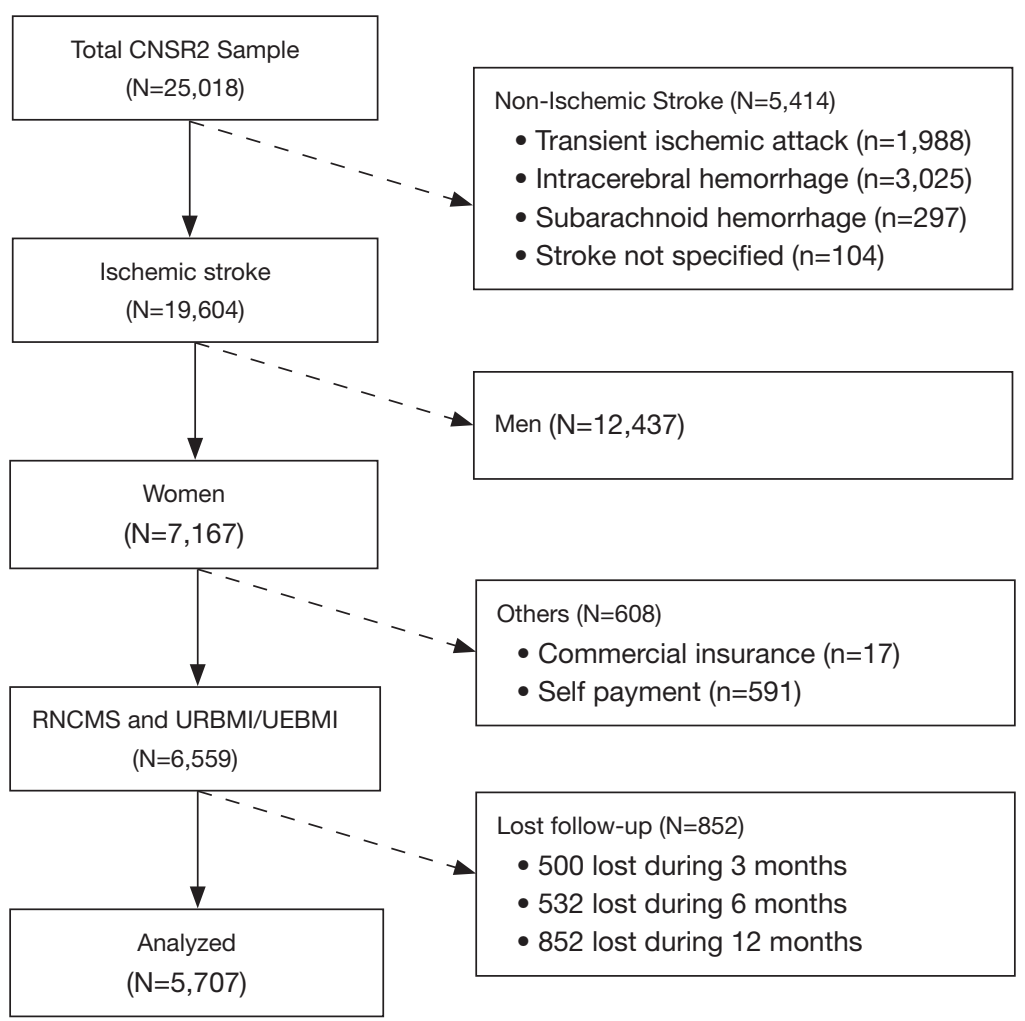

Figure 1 Study flow chart. CNSR II, China National Stroke Registry II; NRCMS, New Rural Cooperative Medical Scheme; URBMI/ UEBMI, urban resident/employee-based basic medical insurance.

on the current literature reviews. The candidate risk factors in our analysis include: age, income, education, NIHSS, mRS score, medical history including (previous stroke/TIA, dyslipidemia, atrial fibrillation, myocardial infarction, heart failure, peripheral vascular disease, coronary heart disease, hypertension disease, diabetes mellitus, smoking status, and alcohol consumption status). Hospitals were integrated into the model calculations using the PHREG Procedure in the SAS (17). To account for hospital clustering and obtain a more precise estimate, shared frailty models were used to generate unadjusted and adjusted models. As hospital-level (secondary $v s$. tertiary hospitals) might be an important confounding factor when assessing the association between insurance status and 1-year outcomes. Therefore, to control the confounding effect, we add hospital level in all the adjusted models.

Similar analysis strategies were performed to investigate the relationship between insurance status and disability. The same set of candidate risk factors was also obtained. To achieve better estimation, generalized estimating equations were applied in both unadjusted and adjusted models to control for the hospital clustering effect (18).

All $\mathrm{P}$ values were 2 -sided, with values of $\mathrm{P}<0.05$ considered to indicate statistical significance. All statistical analyses were performed using SAS Version 9.4 software (SAS Institute, Cary, NC, USA).

\section{Results}

CNSR II enrolled 25,108 acute cerebrovascular diseases patients, among which, 5,414 non-ischemia stroke patients were excluded firstly. Among the remaining 19,604 AIS patients, 7,167 were abstracted. Among these patients, 6,559 $(91.5 \%)$ covered by the NRCMS and URBMI/UEBMI were included. Of these, 5,707 (87.0\%) completed the 1-year follow-up (Figure 1). The baseline characteristics of the women with AIS, compared with those at followup, are shown in Table S2. No significant differences in demographic and clinical characteristics were found except for the NIHSS score on admission, history of alcohol 
consumption, and atrial fibrillation.

\section{Demographics and clinical characteristics}

Table 1 summarizes the characteristics of the 5,707 women with AIS with different rural and urban insurance statuses. Women insured by the NRCMS were younger (mean \pm $\mathrm{SD}, 65.7 \pm 11.4 v s .68 .9 \pm 11.1$ years, $\mathrm{P}<0.0001)$ and had more severe disease statuses (NIHSS score, mean $\pm \mathrm{SD}, 5.8 \pm 5.6$ vs. 5.1 $\pm 5.6, \mathrm{P}<0.0001)$ than those covered by the URBMI/ UEBMI. Compared to women covered by the URBMI/ UEBMI, women covered by the NRCMS were more likely to have a low education level and low household income $(\mathrm{P}<0.0001)$. In addition, compared to women covered by the URBMI/UEBMI, women covered by the NRCMS were less likely to have had a previous stroke $(\mathrm{P}<0.0001)$.

\section{Prevalence, unawareness, and treatment of vascular risk factors before stroke}

For women covered by the NRCMS, the self-reported prevalence rate of coronary heart disease, hypertension, diabetes mellitus, dyslipidemia, atrial fibrillation, peripheral artery disease, and myocardial infarction was lower than that observed for women covered by the URBMI/UEBMI. Women insured by the NRCMS were more likely to demonstrate a higher unawareness rate for hypertension disease $(12 \%$ vs. $8 \%, \mathrm{P}<0.001)$ as well as pre-stroke dyslipidemia $(28 \%$ vs. $23 \%, \mathrm{P}<0.001)$ than those covered by the URBMI/UEBMI. There was no significant difference in the unawareness rates for diabetes mellitus nor for atrial fibrillation between women covered by the NRCMS and those covered by the URBMI/UEBMI (Figure 2).

Overall, the treatment rates of hypertension disease and diabetes mellitus were $>60 \%$; however, the treatment rates for other risk factors ranged from $7 \%$ to $20 \%$. Compared to women covered by the URBMI/UEBMI, women covered by the NRCMS were less likely to use antiplatelet drugs $(22.0 \%$ vs. $17.7 \%, \mathrm{P}<0.0001)$, anti-hypertension medications $(68.3 \%$ vs. $58.1 \%, \mathrm{P}<0.0001)$, lipid-lowering drugs $(22.6 \%$ vs. $14.4 \%, \mathrm{P}<0.0001)$, and anti-diabetic drugs (65.0\% vs. $57.8 \%, \mathrm{P}=0.0019)$ (Table 2).

\section{Quality of stroke care and prevention measures}

Compliance with the usage of the seven evidence-based quality indicators for medication slightly varied between women with rural and those with urban insurance statuses, although with statistical significance. Compared to women covered by the URBMI/UEBMI, women covered by the NRCMS were more likely to receive early antithrombotic treatment upon hospitalization $(83.2 \%$ vs. $85.6 \%)$, antithrombotics at discharge $(88.2 \%$ vs. $91.4 \%)$, lipidlowering drugs for LDL levels $\geq 100 \mathrm{mg} / \mathrm{dL}$ at discharge (63.4\% vs. 71.6\%), but less likely to receive medication for hypertension disease $(67.8 \%$ vs. $64.8 \%$; $\mathrm{P}=0.0286)$. There were no statistically significant differences in the administration of intravenous thrombolytics, anticoagulants for atrial fibrillation, and antidiabetic medications for diabetes mellitus (Table 3).

\section{One-year clinical outcomes}

Among women covered by the NRCMS and URBMI/ UEBMI who experienced AIS, the incidence of allcause death was 558 (9.8\%), stroke recurrence was 302 $(5.3 \%)$, and disability at 1 -year after stroke onset was $1,536(26.9 \%)$. After adjusting for hospital level and other selected covariates, using URBMI/UEBMI status as the reference, the hazard ratio of all-cause death and stroke recurrence after 1 year were significantly higher for women covered by the NRCMS $[10.93 \%$ vs. $8.65 \%$ and $5.77 \%$ vs. $4.83 \%$, respectively; adjusted hazard ratio $(95 \%$ confidence interval): 1.40 (1.06-1.84) and 1.38 (1.04-1.83), respectively] (Table 4). There was no difference in the incidence of disability between women with rural and those with urban insurance status (Table 4).

\section{Discussion}

Female AIS patients covered by the NRCMS were less likely to demonstrate self-reported vascular risk factors and receive pre-stroke treatment for hypertension and dyslipidemia than those by the URBMI/UEBMI; however, these women were more likely to receive medications for acute treatment and secondary prevention during hospitalization. Although the risk-factor burden was lower and the mean age was younger for women covered by the NRCMS, the incidence of 1 -year stroke recurrence and death was higher after adjusting for a series of covariates.

The Prospective Urban Rural Epidemiology (PURE) study also observed the above-mentioned similar result that in middle-income and low-income countries, the allcause mortality rates were significantly higher in rural communities than in urban communities, whereas the risk factor burden was higher in urban communities 
Table 1 Baseline characteristics of women with acute ischemic stroke covered by the URBMI/UEBMI and NRCMS

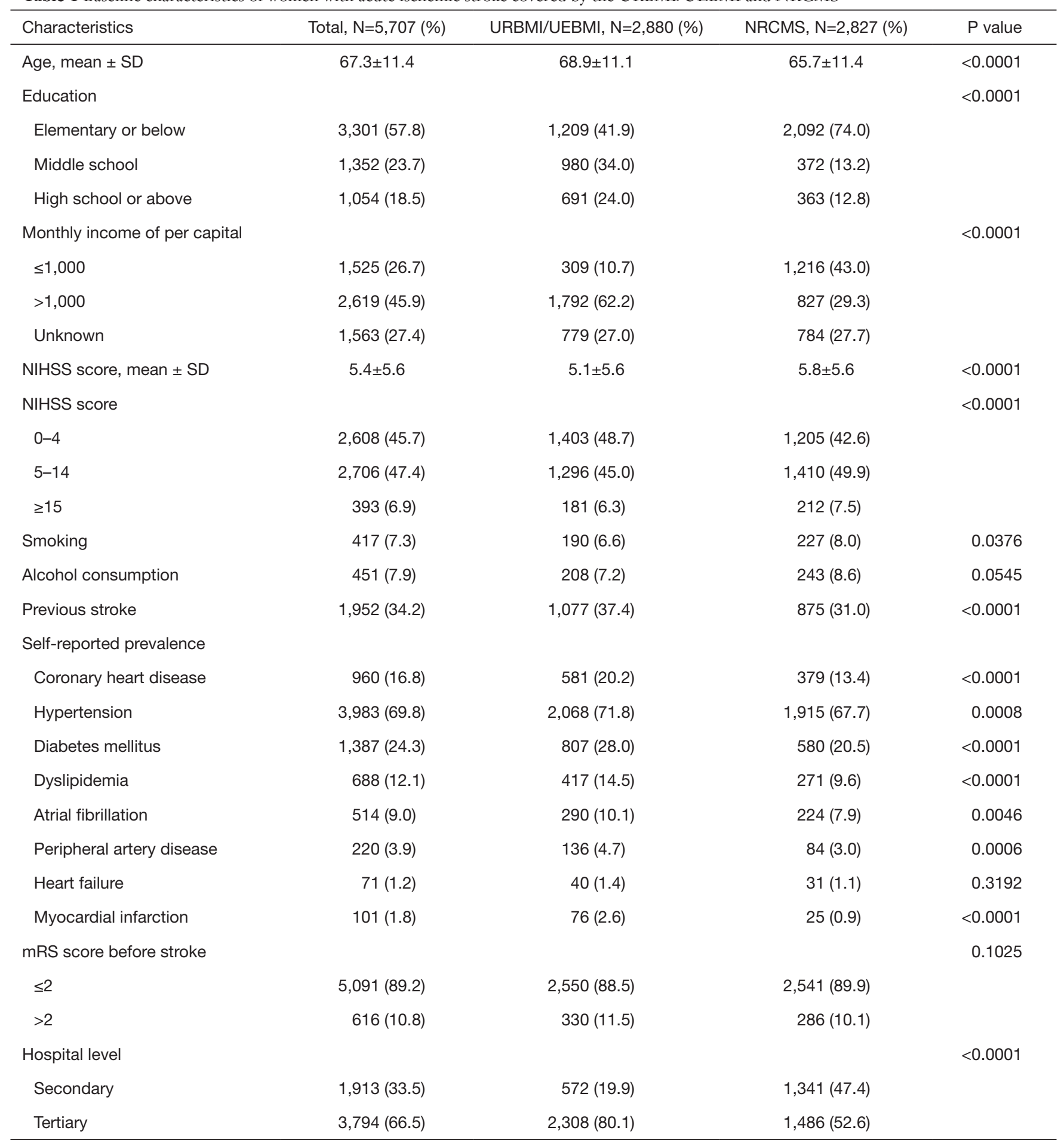

mRS, modified Rankin scale; NIHSS, National Institutes of Health Stroke Scale; NRCMS, New Rural Cooperative Medical Scheme; SD, standard deviation; TIA, transient ischemic attack; URBMI/UEBMI, urban resident/employee basic medical insurance. 


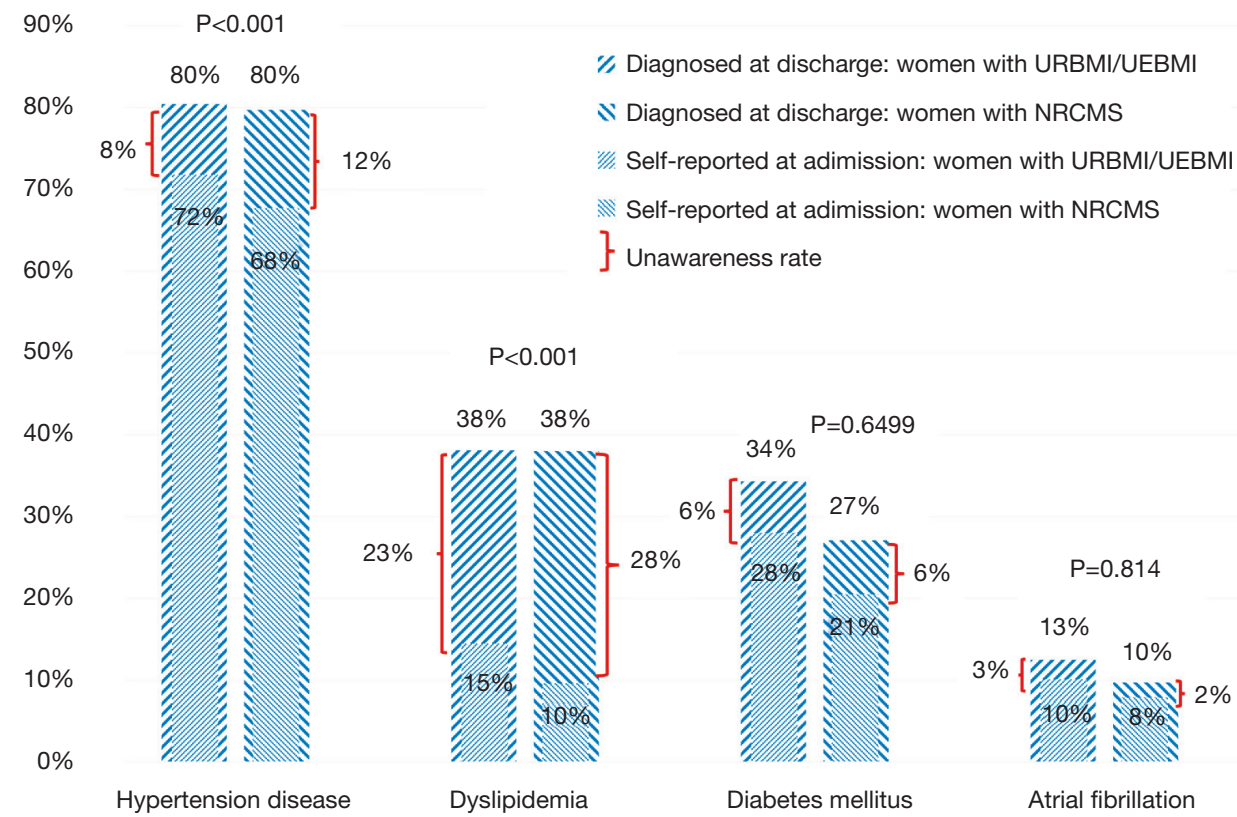

Figure 2 Self-reported prevalence at admission, diagnosed prevalence at discharge, and disease unawareness of vascular risk factors. NRCMS indicates New Rural Cooperative Medical Scheme; and URBMI/UEBMI, Urban Resident/Employee-Based Basic Medical Insurance Scheme. P value was calculated for the comparison of unawareness of these four vascular risk factors between women with URBMI/UEBMI and NRCMS. NRCMS, New Rural Cooperative Medical Scheme; URBMI/UEBMI, urban resident/employee-based basic medical insurance.

Table 2 Treatment of vascular risk factors prior to stroke among women with ischemic stroke covered by the URBMI/UEBMI and NRCMS

\begin{tabular}{|c|c|c|c|c|c|}
\hline Treatment & Eligible population*, N (\%) & \multicolumn{3}{|c|}{ Treatment } & $P$ value \\
\hline Antiplatelet & $5,707(100.0)$ & $1,133(19.9)$ & $633(22.0)$ & $500(17.7)$ & $<0.0001$ \\
\hline Anticoagulation & $634(11.1)$ & $44(6.9)$ & $26(7.2)$ & $18(6.6)$ & 0.7481 \\
\hline Anti-hypertension & $4,568(80.0)$ & 2,891 (63.3) & $1,582(68.3)$ & $1,309(58.1)$ & $<0.0001$ \\
\hline Anti-diabetics & $1,752(30.7)$ & $1,084(61.9)$ & $642(65.0)$ & $442(57.8)$ & 0.0019 \\
\hline
\end{tabular}

*Eligible population indicates that the population is eligible for these drug treatments. NRCMS, New Rural Cooperative Medical Scheme; URBMI/UEBMI, urban resident/employee basic medical insurance.

than in rural communities (19). Improved management of cardiovascular diseases, such as the utilization of preventive medications and revascularization procedures, was significantly more common in high-income countries than in middle-income or low-income countries $(19,20)$. Possible contributing factors could include access to and affordability of health services and medications, thresholds for diagnoses and treatments, and the educational level of the population (20). Additionally, female stroke patients in rural areas had more severe conditions than those in urban areas. A previous study showed that illnesses lasting two weeks were more severe in rural residents than in urban residents (21). Thus, the severity of stroke increases the risk of mortality (22). There may be more considerable differences between urban and rural communities in terms of the access to quality health care and affordability of such care, both of which may contribute to higher rates of mortality due to cardiovascular causes in rural areas, despite 
Table 3 Quality of stroke care among women with ischemic stroke patients covered by the URBMI/UEBMI and NRCMS

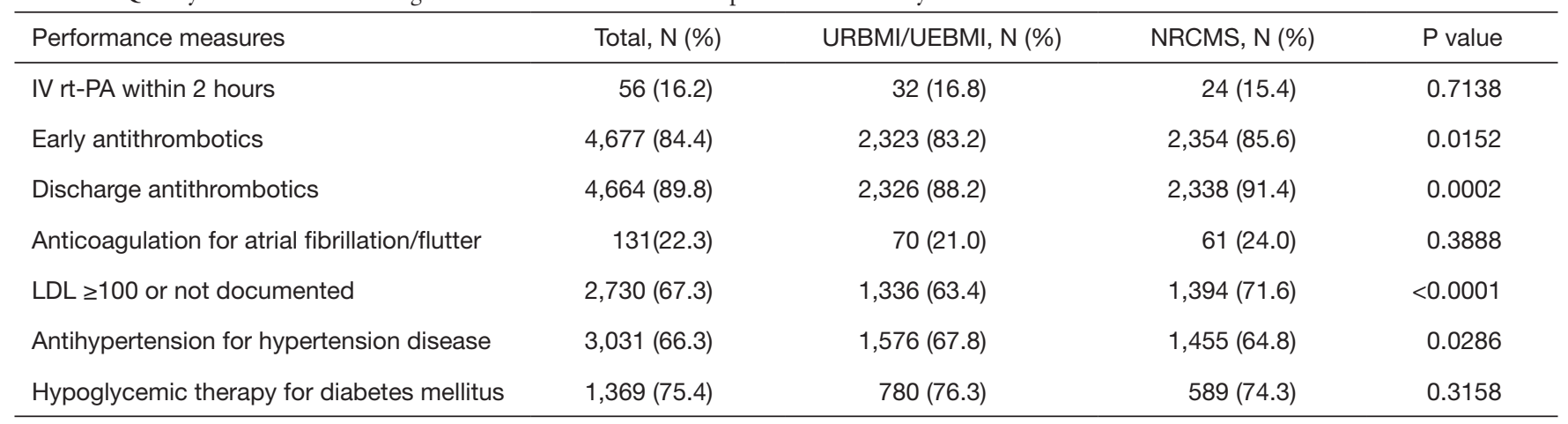

IV rt-PA, intravenous recombinant tissue-type plasminogen activator; LDL, low-density lipoprotein; NRCMS, New Rural Cooperative Medical Scheme; URBMI/UEBMI, urban resident/employee basic medical insurance.

Table 4 Association between risk factors and 1- year outcomes

\begin{tabular}{|c|c|c|c|c|c|}
\hline Variables & 1-year outcomes, n (\%) & \multicolumn{2}{|c|}{ Crude results } & \multicolumn{2}{|c|}{ Adjusted results } \\
\hline \multicolumn{6}{|l|}{ All-cause death } \\
\hline \multicolumn{6}{|l|}{ Insurance status } \\
\hline NRCMS & $309(10.93)$ & 1.42 & $1.11-1.83$ & 1.40 & $1.06-1.84$ \\
\hline \multicolumn{6}{|c|}{ 1-year stroke recurrence } \\
\hline \multicolumn{6}{|l|}{ Insurance status } \\
\hline NRCMS & $163(5.77)$ & 1.26 & $0.98-1.62$ & 1.38 & $1.04-1.83$ \\
\hline URBMI/UEBMI & $139(4.83)$ & Ref & Ref & Ref & Ref \\
\hline \multicolumn{6}{|l|}{ 1-year disability } \\
\hline \multicolumn{6}{|l|}{ Insurance status } \\
\hline NRCMS & $757(26.3)$ & 1.07 & $0.95-1.20$ & 0.92 & $0.79-1.09$ \\
\hline URBMI/UEBMI & $779(27.6)$ & Ref & Ref & Ref & Ref \\
\hline
\end{tabular}

All-cause death and 1-year stroke recurrence used HR to measure associations. 1-year disability used OR to measure associations. For all-cause death, adjusted variables include hospital level, age, income, NIHSS score, mRS score, smoking, atrial fibrillation, and heart failure; for 1-year recurrence, adjusted variables include hospital level, age, income, NIHSS score, mRS score, previous stroke/TIA, coronary heart disease, hypertension, diabetes mellitus, atrial fibrillation and myocardial infarction; for 1-year disability, adjusted variables include hospital level, age, education, NIHSS score, mRS score, previous stroke/TIA, coronary heart disease, diabetes mellitus, atrial fibrillation and heart failure. $\mathrm{HR}$, hazard ratio; OR, odds ratio; $\mathrm{Cl}$, confidence interval.

a lower risk-factor burden (19). In addition, populations living in rural China have limited access to health care compared with urban residents as well as a greater financial burden, mainly because of low funds from the NRCMS (8). In our study, we also found that, compared to women covered by the URBMI/UEBMI, female AIS patients covered by the NRCMS had a lower household income and education level. Contributions of total funds toward household expenditures for health care for urban and rural households were $5 \%$ and $8 \%$, respectively (23). A considerable and increasing disparity in the disbursement of health care funding exists between urban and rural regions. This health care financing inequality should be given more attention (23). 
Female AIS patients covered by the NRCMS were less frequently aware of and less frequently received treatment for stroke vascular risk factors such as hypertension and dyslipidemia than those covered by the URBMI/UEBMI. These findings are consistent with several previous global and national studies (24-26). The PURE study showed that the awareness, treatment, and control of hypertension were all lower in rural communities in the middle- or low-income countries $(24,25)$. In the Beijing eye study, the awareness, treatment, and control of hypertension were all lower in rural than in urban residents (27).

We also found that the utilization of primary preventive healthcare treatments such as antiplatelet, lipid-lowering, antihypertensive, and antidiabetic drugs was low in female AIS patients covered by the NRCMS. The representative sample data from the China Health and Nutrition Survey in 2011 showed that rural residents were less likely to utilize preventive care services than urban residents $(5.1 \%$ vs. $9.3 \%$ ) (28). Secondary prevention medication for cardiovascular disease may be unavailable or unaffordable for a large proportion of communities and households. This is more evident in rural communities than in urban communities (26). These reasons could partly explain the poorer treatment and control rates among individuals from rural communities, for whom limited drug availability and affordability can be particularly disadvantageous. The effects of community-based standardized management of hypertension were more evident in urban areas than in rural areas. The quality of service for community-based hypertension management in rural areas was found to be lower than in urban areas (29). This is likely a reflection of a combination of factors related to low socioeconomic status and may be influenced by a lack of access to care, lack of knowledge of the sequelae of uncontrolled stroke risk factors, and differing values with respect to the importance of the future.

As for the quality of stroke care and prevention, some interesting findings from this study include the higher likelihood of early antithrombotic treatment during hospitalization, antithrombotics at discharge, and lipidlowering treatment for $\mathrm{LDL} \geq 100 \mathrm{mg} / \mathrm{dL}$ at discharge among female AIS patients covered by the NRCMS; however, in contrast, these women were less likely to be prescribed antihypertensive medication than those covered by the URBMI/UEBMI. Previous studies also observed differences in the process of care among patients (30). In our study, the participating hospitals randomly received patients with a rural or urban insurance status and provided similar stroke care service. These medications for stroke care and prevention are covered by the NRCMS and URBMI/UEBMI. Meanwhile, female AIS patients with NRCMS were younger and had more serious conditions, which may affect the adherence to performance measures of stroke care $(10,13,31)$. Patients covered by the NRCMS who receive medication at outpatient clinics cannot be reimbursed (8); therefore, they prefer to be prescribed treatment at discharge. The expense of these drugs can be covered by the NRCMS.

Since the pilot programs were initiated in 2003, and by 2008 , coverage by these programs was comprehensive. The NRCMS is very close to universal coverage. The participation number of NRCMS has grown steadily every year, from 80 million in the early stage of the pilot programs to 812 million by the end of June 2012, with over $95 \%$ of the targeted rural population covered (7). The data from CNSR I and II demonstrated that the proportion of those individuals with NRCMS increased significantly from $16.8 \%$ in $2007-2008$ to $39.5 \%$ in $2012-2013$ (10). However, rural residents are still underutilizing preventive primary healthcare services, when compared to their urban counterparts (28). In CNSR II, we found the quality of the process of stroke care during hospitalization for women covered by the NRCMS to be equal to, or superior to that received by women covered by the URBMI/UEBMI. Meanwhile, our study indicated that the heavier burden of stroke in women has shifted from urban to rural regions where a larger population dwells, and where healthcare service is less developed than in urban regions in China (32).

However, there are several limitations in our study. First, even though we adjusted for several important covariates, unmeasured and residual confounding is possible. We did not have information on the patients' employment or detailed lifestyle data, nor access to data regarding patient usage of healthcare services prior to the stroke and after discharge. These factors may affect patient clinical outcomes. Second, hospitals participating in the CNSR II may be different from other hospitals. There are differences in terms of medical skills and medical facilities between secondary and tertiary hospitals. The sampling schema we used was not a strictly multi-stage random nationwide sampling approach. We used the shared frailty model and generalized estimating equation model, after accounting for the cluster effect of hospitals, for more precise estimation. However, caution is needed when interpreting the data as representative of female AIS patients with urban or rural insurance statuses. Third, although it would have been 
preferable to have obtained information on the reasons for the poorer outcome among female AIS patients covered by the NRCMS, detailed information was not available at this time. Nonetheless, the causes of these associations remain unclear. The process of care after discharge should be further explored.

\section{Conclusions}

Using our large data set of female patients with AIS, we found significant differences in age and stroke risk factor awareness among patients, as well as in the management of these factors, process of care, and 1-year stroke recurrence rates and mortality associated with rural and urban insurance status. Our data demonstrate that women covered by the NRCMS are associated with worse long-term clinical outcomes after stroke. The heavier burden of stroke among women has shifted from urban to rural regions. Healthcare policymakers need to focus their attention on these disparities and take the necessary steps to improve primary healthcare services in rural areas.

\section{Acknowledgments}

We thank all participating hospitals, colleagues, nurses, imaging and laboratory technicians, and the Chinese National Stroke Registry Steering Committee members.

Funding: This work was supported by grants from National Key R\&D Program of China (2017YFC1310901, 2016YFC0901002, 2016YFC0901001), Beijing Municipal Committee of Science and Technology (D151100002015003), Beijing Municipal Administration of Hospitals' Mission Plan (SML20150502).

\section{Footnote}

Conflicts of Interest: The authors declare they have no conflict of interests.

Ethical Statement: The authors are accountable for all aspects of the work in ensuring that questions related to the accuracy or integrity of any part of the work are appropriately investigated and resolved. The study was approved by the Central Institutional Review Board at Beijing Tiantan Hospital (ky2012-005-01) and written informed consent was obtained from all patients.

\section{References}

1. Bushnell C, McCullough LD, Awad IA, et al. Guidelines for the prevention of stroke in women: a statement for healthcare professionals from the American Heart Association/American Stroke Association. Stroke 2014;45:1545-88.

2. He J, Gu D, Wu X, et al. Major causes of death among men and women in China. N Engl J Med 2005;353:1124-34.

3. Wang J, Ning X, Yang L, et al. Sex differences in trends of incidence and mortality of first-ever stroke in rural Tianjin, China, from 1992 to 2012. Stroke 2014;45:1626-31.

4. National Health Commission of the People's Republic of China. China's Health Statistics Yearbook 2010. Beijing: Peking Union Medical College Press, 2010.

5. Chen WW, Gao RL, Liu LS, et al. China cardiovascular diseases report 2015: A summary. J Geriatr Cardiol 2017;14:1-10.

6. Reeves MJ, Bushnell CD, Howard G, et al. Sex differences in stroke: epidemiology, clinical presentation, medical care, and outcomes. Lancet Neurol 2008;7:915-26.

7. National Health Commission of the People's Republic of China. The Development of China's New Rural Cooperative Medical Scheme. Http://www.Moh.Gov.Cn/ mohbgt/s3582/201209/55893.Shtml. Accessed [Date (May 15, 2019)].

8. Meng Q, Fang H, Liu X, et al. Consolidating the social health insurance schemes in China: towards an equitable and efficient health system. Lancet 2015;386:1484-92.

9. Yang X, Li Z, Zhao X, et al. Use of Warfarin at Discharge Among Acute Ischemic Stroke Patients with Nonvalvular Atrial Fibrillation in China. Stroke 2016;47:464-70.

10. Li Z, Wang C, Zhao X, et al. Substantial progress yet significant opportunity for improvement in stroke care in china. Stroke 2016;47:2843-9.

11. Rankin J. Cerebral Vascular Accidents in Patients over the Age of 60: II. Prognosis. Scot Med J 1957;2:200-15.

12. Lyden P, Brott T, Tilley B, et al. Improved reliability of the NIH Stroke Scale using video training. NINDS TPA Stroke Study Group. Stroke 1994;25:2220-6.

13. Schwamm LH, Fonarow GC, Reeves MJ, et al. Get With the Guidelines-Stroke is associated with sustained improvement in care for patients hospitalized with acute stroke or transient ischemic attack. Circulation 2009;119:107-15. 
14. Neurology Branch of Chinese Medical Association Group of Cerebrovascular Disease. 2010 Chinese guidelines for diagnosis and treatment of acute ischemic stroke. Chin J Neurol 2010;43:146-53.

15. Fonarow GC, Reeves MJ, Smith EE, et al;GWTG-Stroke Steering Committee and Investigators. Characteristics, performance measures, and in-hospital outcomes of the first one million stroke and transient ischemic attack admissions in Get With The Guidelines-Stroke. Circ Cardiovasc Qual Outcomes 2010;3:291-302.

16. National Health Commission of the People's Republic of China. China Health Statistics Yearbook 2009. Beijing: Peking Union Medical College Press, 2009.

17. Wienke A. Frailty Models in Survival Analysis. Boca Raton: CRC Press, 2010.

18. Hardin J. Generalized Estimating Equations (GEE). Wiley Online Library, 2005.

19. Yusuf S, Rangarajan S, Teo K, et al. Cardiovascular risk and events in 17 low-, middle-, and high-income countries. N Engl J Med 2014;371:818-27.

20. O'Flaherty M, Buchan I, Capewell S. Contributions of treatment and lifestyle to declining CVD mortality: why have CVD mortality rates declined so much since the 1960s? Heart 2013;99:159-62.

21. Tian D, Sun L, Zhang L, et al. Large urban-rural disparity in the severity of two-week illness: updated results based on the first health service survey of Hunan Province, China. Int J Equity Health 2016;15:37.

22. Zhang N, Liu G, Zhang G, et al. External validation of the iScore for predicting ischemic stroke mortality in patients in China. Stroke 2013;44:1924-9.

23. Wang L, Wang A, Zhou D, et al. An Empirical Analysis of Rural-Urban Differences in Out-Of-Pocket Health Expenditures in a Low-Income Society of China. PloS one 2016;11:e0154563.

24. Aune D, Keum N, Giovannucci E, et al. Nut consumption and risk of cardiovascular disease, total cancer, all-cause and cause-specific mortality: a systematic review and dose- response meta-analysis of prospective studies. BMC Med 2016;14:207.

25. Chow CK, Teo KK, Rangarajan S, et al. Prevalence, awareness, treatment, and control of hypertension in rural and urban communities in high-, middle-, and low-income countries. JAMA 2013;310:959-68.

26. Khatib R, McKee M, Shannon H, et al. Availability and affordability of cardiovascular disease medicines and their effect on use in high-income, middle-income, and lowincome countries: an analysis of the PURE study data. Lancet 2016;387:61-9.

27. Xu L, Wang S, Wang YX, et al. Prevalence of arterial hypertension in the adult population in rural and urban China: the Beijing eye study. Am J Hypertens 2008;21:1117-23.

28. Liu X, Li N, Liu C, et al. Urban-rural disparity in utilization of preventive care services in China. Medicine 2016;95:e4783.

29. Zeng XY, Zhang M, Li YC, et al. Study on effects of community-based management of hypertension patients aged $\geq 35$ years and influencing factors in urban and rural areas of China, 2010. Zhonghua Liu Xing Bing Xue Za Zhi 2016;37:612-7.

30. James ML, Grau-Sepulveda MV, Olson DM, et al. Insurance status and outcome after intracerebral hemorrhage: findings from Get With The Guidelinesstroke. J Stroke Cerebrovasc Dis 2014;23:283-92.

31. Katzan IL, Spertus J, Bettger JP, et al. Risk adjustment of ischemic stroke outcomes for comparing hospital performance: a statement for healthcare professionals from the American Heart Association/American Stroke Association. Stroke 2014;45:918-44.

32. Zhang XH, Guan T, Mao J, et al. Disparity and its time trends in stroke mortality between urban and rural populations in China 1987 to 2001: changing patterns and their implications for public health policy. Stroke 2007;38:3139-44.
Cite this article as: Gu HQ, Yang X, Rao ZZ, Wang CJ, Zhao XQ, Wang YL, Liu LP, Liu C, Li H, Li ZX, Wang YJ; on behalf of China National Stroke Registries Investigators. Disparities in outcomes associated with rural-urban insurance status in China among inpatient women with stroke: a registrybased cohort study. Ann Transl Med 2019;7(18):426. doi: 10.21037/atm.2019.08.125 
Supplementary

Table S1 Specifications of guideline-recommended performance measures

Performance measure of ischemic stroke care Performance measure definition for eligible patients*

IV rt-PA within 2 hours

Patients who received IV rt-PA within 2 hours after the initial symptom onset and were treated within 3 hours

Early antithrombotics

Antithrombotic therapy prescribed within 48 hours of hospitalization, including antiplatelet or anticoagulant therapy

Discharge antithrombotics

Antithrombotic therapy prescribed at discharge

Anticoagulation for atrial fibrillation/flutter

Anticoagulation therapy prescribed at discharge for patients with atrial fibrillation or atrial flutter documented during hospitalization

LDL $\geq 100$ or not documented

Lipid-lowering agent prescribed at discharge if the LDL level was $\geq 100 \mathrm{mg} / \mathrm{dL}$, if the patient received lipid-lowering agents prior to admission, or if the LDL level was not documented

Antihypertensives for hypertension disease

Antihypertension medication prescribed at discharge for patients with a history of hypertension disease or hypertension disease documented during hospitalization

Hypoglycemic therapy for diabetes mellitus:

Hypoglycemic medication prescribed at discharge for patients with a history of diabetes mellitus or diabetes mellitus documented during hospitalization

*Eligible patients include only those without any medical contraindications (e.g., treatment intolerance, excessive risk for adverse reaction, patient/family refusal, or terminal illness/comfort care only) documented as reasons for non-treatment for each of the applicable measures. This excludes patients who are discharged to hospice, or another short-term general hospital and those who decided to leave against medical advice before the end of hospital day two. Early antithrombotics excludes patients who died before the end of hospital day two. The acute rt-PA measure excludes patients with missing or erroneous onset arrival or treatment times; and who began IV rt-PA treatment at an outside hospital; and those who initiated IV rt-PA treatment after 180 minutes from onset. Performance measures at discharge, excludes patients who died during hospitalization. We employed the same criteria for the five performance measures as the Get With The Guideline-Stroke. IV rt-PA, intravenous recombinant tissue plasminogen activator; LDL, low-density lipoprotein. 
Table S2 Characteristics of the analyzed and patient populations

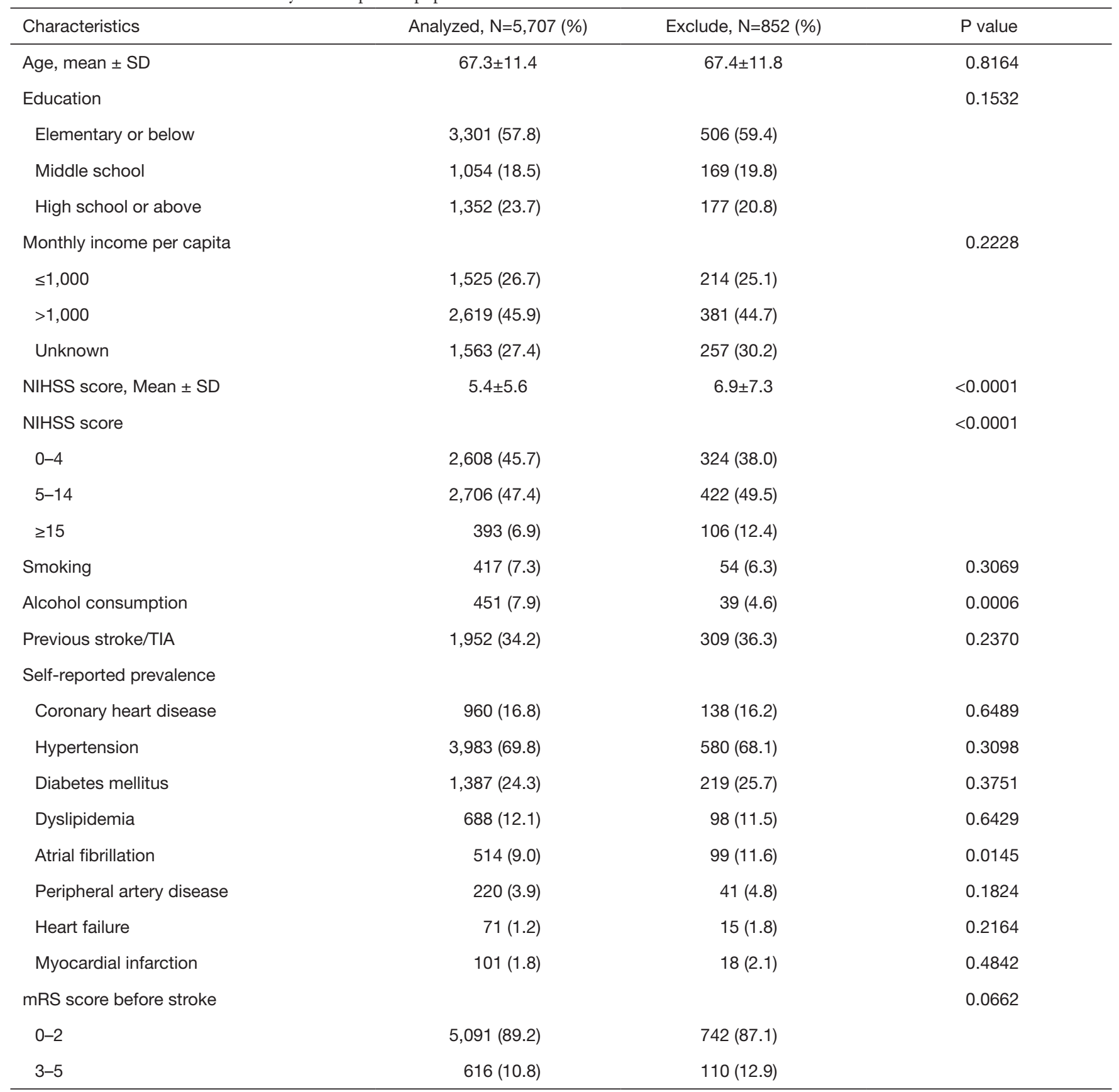

mRS, modified Rankin Scale; NIHSS, National Institutes of Health Stroke Scale; NRCMS, New Rural Cooperative Medical Scheme; SD, standard deviation; TIA, transient ischemic attack; URBMI/UEBMI, urban resident/employee basic medical insurance. 